Post-print of: Journal of Mathematical Economics, 49(3), 2013, 222-229

\title{
Local and Global Consistency Properties for Student Placement*
}

\author{
Bettina Klaus ${ }^{\dagger} \quad$ Flip Klijn ${ }^{\ddagger}$
}

January 2013

\begin{abstract}
In the context of resource allocation on the basis of priorities, Ergin (2002) identifies a necessary and sufficient condition on the priority structure such that the student-optimal stable mechanism satisfies a consistency principle. Ergin (2002) formulates consistency as a local property based on a fixed population of agents and fixed resources - we refer to this condition as local consistency and to his condition on the priority structure as local acyclicity. A related but stronger necessary and sufficient condition on the priority structure such that the student-optimal stable mechanism satisfies a more standard global consistency property is unit acyclicity.

We provide necessary and sufficient conditions for the student-optimal stable mechanism to satisfy converse consistency principles. First, we identify a necessary and sufficient condition (local shift-freeness) on the priority structure such that the student-optimal stable mechanism satisfies local converse consistency. Interestingly, local acyclicity implies local shift-freeness and hence the student-optimal stable mechanism more frequently satisfies local converse consistency than local consistency. Second, in order for the student-optimal stable mechanism to be globally conversely consistent, one again has to impose unit acyclicity on the priority structure. Hence, unit acyclicity is a necessary and sufficient condition on the priority structure for the student-optimal stable mechanism to satisfy global consistency or global converse consistency.
\end{abstract}

JEL classification: D63, C78.

Keywords: student placement, consistency, converse consistency, priority structure.

\section{Introduction}

A student placement problem is determined by a set of students, a set of position types, the number of available positions - the quota - of each type, and the students' strict preferences over

\footnotetext{
*Bettina Klaus gratefully acknowledges financial support from the Netherlands Organisation for Scientific Research (NWO) under grant VIDI-452-06-013. Flip Klijn gratefully acknowledges support from Plan Nacional I+D+I (ECO2011-29847). A first draft of this paper was written while Flip Klijn was visiting Harvard Business School. He gratefully acknowledges a research fellowship from HBS.

${ }^{\dagger}$ Faculty of Business and Economics, University of Lausanne, Internef 538, CH-1015 Lausanne, Switzerland; e-mail: bettina.klaus@unil.ch

${ }^{\ddagger}$ Corresponding author: Institute for Economic Analysis (CSIC) and Barcelona GSE, Campus UAB, 08193 Bellaterra (Barcelona), Spain; e-mail: flip.klijn@iae.csic.es
} 
position types (e.g., a position type could represent the admission to a college or university) and remaining unassigned. A student placement mechanism assigns to any given student placement problem an allocation of the position types to the students such that every student receives at most one position and quotas are binding. In contrast to so-called house allocation problems, where an assignment is made on the basis of students' preferences over position types alone, ${ }^{1}$ we assume that in a student placement problem additional information is available. ${ }^{2}$ For instance, college admissions of undergraduate students are often based on rankings obtained from one or several entrance exams. Then, students who achieved higher test scores in the entrance exam of a certain college have higher priority for admission at that college than students with lower test scores. We will model this situation using strict priority rankings of individuals for each position type (possibly using tie-breaking). We call the collection of strict priority rankings a priority structure.

A placement mechanism violates the priority of student $i$ for position $x$ if there exist preferences under which student $i$ envies student $j$ who obtains $x$ even though $i$ has a higher priority for $x$ than $j$. A placement mechanism is fair if it never violates the priority of any student. Ergin (2002) focuses on the so-called student-optimal stable mechanism (introduced by Gale and Shapley, 1962) since it is fair and Pareto superior to any other fair placement mechanism. Ergin (2002, Theorem 1) provides a necessary and sufficient "acyclicity" condition on the priority structure for the student-optimal stable mechanism to satisfy several appealing properties. In particular, he considers a notion of consistency. Loosely speaking, a placement mechanism is consistent if, whenever some students leave with their allotments, the placement mechanism allocates the remaining positions among the students who did not leave in the same way as in the original placement problem. Ergin (2002) formulates consistency as a local property based on a fixed population of agents and fixed resources - we refer to this condition as local consistency and to his condition on the priority structure as local acyclicity. Interestingly, a closely related local consistency property is used by Kojima and Ünver (2011) to characterize the so-called "Boston" mechanism, which is a direct competitor of the student-optimal stable mechanism in the context of school choice.

The local consistency notions, however, are different from the standard consistency notion since the set of students and the quotas are fixed. A related but stronger necessary and sufficient condition on the priority structure such that the student-optimal stable mechanism satisfies a more standard global consistency property is unit acyclicity (Corollary 1). ${ }^{3}$

The main part of this paper focuses on a property that is closely related to consistency, namely converse consistency. Converse consistency refers to an inverse of the reduction operation that consistency uses. Thomson (2009, page 30) describes converse consistency as a property of "decentralizability:" given some problem, if an allocation is chosen for each of its associated reduced two-agent problems, then it should be chosen for the problem involving the whole group. Converse consistency has practical appeal whenever small problems are much easier to

\footnotetext{
${ }^{1}$ Sometimes it is also assumed that exactly one position of each type is available. Some recent articles on house allocation problems are Ergin (2000), Ehlers (2002), Ehlers et al. (2002), Ehlers and Klaus (2003, 2006, 2007), and Velez (2012).

${ }^{2}$ See, for instance, Balinski and Sönmez (1999), Ergin (2002), and Kesten (2006).

${ }^{3}$ For instance, Thomson's (2009, page 16) "Fundamental Definition" of consistency deals with a variable population setup and imposes the consistency requirement on all subpopulations as well.
} 
solve than large ones. For two-sided matching problems, the two-agent subgroup assumption that converse consistency is based on is usually adjusted to include somewhat larger groups of agents (Thomson, 2009, page 209). Two papers exploring aspects of converse consistency for marriage problems (one-to-one matching problems) are Sasaki and Toda (1992) and ÖzkalSanver (2013). Sasaki and Toda (1992) show that the core correspondence for marriage markets satisfies converse consistency and that this property is part of a core characterization; we briefly discuss Özkal-Sanver (2013) below. For an overview of the literature on converse consistency and its computational implications in other contexts we refer to Thomson $(2009,2011,2012)$.

Özkal-Sanver (2013, Example 2.1) shows that, depending on the priority structure, the student-optimal stable mechanism may not satisfy converse consistency. In view of this negative result, there are (at least) two ways to proceed. The first approach is to expand the studentoptimal stable mechanism to obtain a conversely consistent (multi-valued) correspondence. A particularly interesting correspondence is the minimal expansion that is conversely consistent. Özkal-Sanver (2013) takes this approach and her main result is the identification of the minimal conversely consistent extension of the student-optimal stable mechanism (Özkal-Sanver, 2013, Theorem 3.1). In view of the practical and theoretical relevance of the student-optimal stable matching mechanism, a second approach would consist of the identification of conditions under which it is conversely consistent. Our paper takes this approach. Moreover, in contrast to Özkal-Sanver (2013) (and Sasaki and Toda, 1992), we consider a many-to-one model.

Similarly to the previous discussion on consistency, one can consider local converse consistency based on a fixed set of students and a fixed quota vector, or allow for the general variable population and resource context and consider (standard) global converse consistency. We first identify a necessary and sufficient condition (local shift-freeness) on the priority structure such that the student-optimal stable mechanism satisfies local converse consistency (Theorem 2). Interestingly, local acyclicity implies local shift-freeness (Lemma 2) and hence the student-optimal stable mechanism more frequently satisfies local converse consistency than local consistency (Corollary 2). Furthermore, in situations where at most one position per position type is available, both conditions coincide (Lemma 3) and the student-optimal stable mechanism satisfies local converse consistency if and only if it satisfies local consistency (Corollary 3 ).

Finally, in order for the student-optimal stable mechanism to be globally conversely consistent, one again has to impose unit acyclicity on the priority structure (Corollary 4). Hence, the student-optimal stable mechanism is globally conversely consistent if and only if it is globally consistent (Corollary 5). This result is interesting in view of a result by Velez (2012). In the context of house allocation problems, he shows that there is no logical relation between consistency and converse consistency.

The paper is organized as follows. In Section 2 we introduce the student placement model and the student-optimal stable mechanism. Section 3 (4) contains the local and global (converse) consistency results mentioned above. 


\section{Student Placement}

Let $\bar{N}=\{1, \ldots, n\}$ denote a set of students with $n \geq 3$. Let $X=\left\{x_{1}, \ldots, x_{p}\right\}$ denote a set of (real) position types with $|X| \geq 3 .{ }^{4}$ For each position type $x \in X$, at most $\bar{q}_{x} \in \mathbb{N}$ copies are available with $1 \leq \bar{q}_{x} \leq|\bar{N}|$. Furthermore, while $\bar{q}_{x}$ denotes the maximal number of positions of type $x$ that might become available, by $q_{x} \in\left\{0,1, \ldots, \bar{q}_{x}\right\}$ we denote the number of positions, the quota, of position type $x$ that are available. A quota vector $q \equiv\left(q_{x}\right)_{x \in X}$ denotes the quota of all position types. Note that we use the term "position $x$ " when we refer to one of the $q_{x}$ positions of position type $x$. Let 0 denote the null position, which does not belong to $X$; "receiving the null position" means "not receiving any position." Since the null position is freely available, we simply assume $q_{0}=\infty$.

Each student $i \in \bar{N}$ is equipped with a strict, transitive, and complete preference relation $R_{i}$ over $X \cup\{0\}$, i.e., $R_{i}$ is a linear order over $X \cup\{0\}$. Given $x, y \in X \cup\{0\}, x P_{i} y$ means that student $i$ strictly prefers $x$ to $y$. If $x P_{i} 0$, then position $x$ is acceptable for student $i$, otherwise it is unacceptable (and $0 P_{i} x$ ). Let $\mathcal{R}$ denote the set of strict, transitive, and complete preference relations over $X \cup\{0\}$. For each $N \subseteq \bar{N}, \mathcal{R}^{N}$ is the set of (preference) profiles $R=\left(R_{i}\right)_{i \in N}$ such that for all $i \in N, R_{i} \in \mathcal{R}$. Given $N^{\prime} \subseteq N \subseteq \bar{N}$ and $R \in \mathcal{R}^{N}$, let $R_{N^{\prime}}$ denote the profile $\left(R_{i}\right)_{i \in N^{\prime}}$; it is the restriction of profile $R$ to the set of students $N^{\prime}$.

Let $x \in X$. We call a linear order $\succ_{x}$ over $\bar{N}$ a priority ordering for position type $x$. Given $i, j \in \bar{N}, i \neq j$, student $i$ has a higher priority for position $x$ than student $j$ if $i \succ_{x} j$. A priority structure is a profile $\succ=\left(\succ_{x}\right)_{x \in X}$ specifying for each position type a priority ordering.

A (student) placement problem $(N, R, q)$ consists of a (finite) set of students $N \subseteq \bar{N}$, preferences $R \in \mathcal{R}^{N}$, and a quota vector $q=\left(q_{x}\right)_{x \in X}$ such that for all positions $x \in X, 0 \leq q_{x} \leq \bar{q}_{x}$. We assume that the null position is available in any placement problem. Finally, we assume that a priority structure $\succ$ is externally given (we do not include it in the description of a placement problem because we assume it to be fixed).

For each placement problem $(N, R, q)$, each student $i \in N$ is to be allocated exactly one position in $X \cup\{0\}$ taking quotas as upper bounds. Formally, an allocation for $(N, R, q)$ is a list $\alpha=\left(\alpha_{i}\right)_{i \in N}$ such that for all $i \in N, \alpha_{i} \in X \cup\{0\}$, and for all $x \in X,\left|\left\{i \in N: \alpha_{i}=x\right\}\right| \leq q_{x}$. Thus, by definition an allocation respects the quotas. Note that not all available positions need to be assigned. Given $i \in N$, we call $\alpha_{i}$ the allotment of student $i$ at $\alpha$.

Next, we introduce the notion of a reduced student placement problem and of a reduced allocation. Consider a student placement problem $(N, R, q)$, an allocation $\alpha$ for it, and a subset $N^{\prime} \subseteq N$ of students. Then, the reduced placement problem $\left(N^{\prime}, R_{N^{\prime}}, q\left(N^{\prime}, \alpha\right)\right)$ for students $N^{\prime}$ at allocation $\alpha$ is defined as the placement problem where the set of students equals $N^{\prime}$ and the only position types that are available to them are those not allocated to students in $N \backslash N^{\prime}$ at $\alpha$, i.e., for all $x \in X, q\left(N^{\prime}, \alpha\right)_{x}=q_{x}-\left|\left\{i \in N \backslash N^{\prime}: \alpha_{i}=x\right\}\right|$. Let $\alpha_{N^{\prime}}$ denote the allocation $\left(\alpha_{i}\right)_{i \in N^{\prime}}$. It is the restriction of allocation $\alpha$ to the set of students $N^{\prime}$. Note that $\alpha_{N^{\prime}}$ is an allocation for $\left(N^{\prime}, R_{N^{\prime}}, q\left(N^{\prime}, \alpha\right)\right)$.

\footnotetext{
${ }^{4}$ The cases $|\bar{N}| \leq 2$ or $|X| \leq 2$ are trivial because then the central properties of the article (consistency and converse consistency) have no bite. Furthermore, our results remain unchanged for infinite $\bar{N}$ or $X$.
} 
An allocation $\alpha$ is individually rational for placement problem $(N, R, q)$ if for each $i \in N$, allotment $\alpha_{i}$ is acceptable for student $i$.

An allocation $\alpha$ is non-wasteful for placement problem $(N, R, q)$ if there are no student $i \in N$ and position $x \in X$ such that $x P_{i} \alpha_{i}$ and $\left|\left\{j \in N: \alpha_{j}=x\right\}\right|<q_{x}$.

An allocation $\alpha$ violates the priority of student $i \in N$ for placement problem $(N, R, q)$ if there exists a position $x$ such that student $i$ has a higher priority for $x$ than one of the students assigned to it and student $i$ prefers to switch to position $x$, i.e., there exist $x \in X$ and $j \in N \backslash\{i\}$ such that $i \succ_{x} j, \alpha_{j}=x$, and $x P_{i} \alpha_{i}$.

A (student) placement mechanism is a function $\varphi$ that assigns to each placement problem $(N, R, q)$ an allocation $\varphi(N, R, q)$.

A placement mechanism $\varphi$ is individually rational if for each placement problem $(N, R, q)$, $\varphi(N, R, q)$ is individually rational for placement problem $(N, R, q)$.

A placement mechanism $\varphi$ is non-wasteful if for each placement problem $(N, R, q), \varphi(N, R, q)$ is non-wasteful for placement problem $(N, R, q)$.

A placement mechanism $\varphi$ is fair (Balinski and Sönmez, 1999) if for each placement problem $(N, R, q), \varphi(N, R, q)$ does not violate the priority of any student for placement problem $(N, R, q)$.

Given a placement problem $(N, R, q)$, we can associate $(N, R, q)$ with a college admissions problem as follows (Balinski and Sönmez, 1999): the set of students equals $N$, the set of position types $X \cup\{0\}$ corresponds to the set of colleges, the quota vector $q$ describes colleges' quotas, preferences $R$ correspond to students' preferences over colleges, and the priority structure $\succ$ is taken to represent colleges' responsive preferences over students with the additional specification that the priority ordering $\succ_{0}$ of the "null college" can be arbitrary. Furthermore (Balinski and Sönmez, 1999, Lemma 2), an allocation $\alpha$ is individually rational, non-wasteful, and fair for placement problem $(N, R, q)$ if and only if the associated "matching" $\alpha$ is stable for the associated college admissions problem, i.e., $\alpha$ is individually rational for $(N, R, q)$ and there exists no student-position blocking pair $(i, x) \in N \times(X \cup\{0\})$ such that $x P_{i} \alpha_{i}$ and (s1) $\mid\{j \in$ $\left.N: \alpha_{j}=x\right\} \mid<q_{x}$ or (s2) there exists $k \in N$ such that $\alpha_{k}=x$ and $i \succ_{x} k{ }^{5}$

For each placement problem $(N, R, q)$, we denote by $\varphi^{\succ}(N, R, q)$ the student-optimal stable allocation for placement problem $(N, R, q)$ that is obtained by using Gale and Shapley's (1962) student-proposing deferred-acceptance algorithm:

- At the first step of the student-proposing deferred-acceptance algorithm, every student in $N$ applies to her/his favorite position. For each position $x \in X \cup\{0\}$, the $q_{x}$ applicants who have the highest priority for $x$ (all applicants if there are fewer than $q_{x}$ or $x=0$ ) are placed on the waiting list of position $x$, and all others are rejected. (If $q_{x}=0$, then all proposing students are rejected.)

- At the $r$-th step of the student-proposing deferred-acceptance algorithm, those applicants who were rejected at step $r-1$ apply to their next best position. For each position

\footnotetext{
${ }^{5}$ The definition of stability here is less general than the one for college admissions problems because for student placement problems, position types always "find all students acceptable." For more details on the well-known college admissions model and basic and well-known results for this model, we refer the interested reader to Gale and Shapley (1962) and Roth and Sotomayor (1990).
} 
$x \in X \cup\{0\}$, the $q_{x}$ applicants among the new applicants and those on the waiting list who have the highest priority for position $x$ are placed on the updated waiting list of position $x$, and all others are rejected.

The student-optimal deferred-acceptance algorithm terminates when every student is on a waiting list. Note that the null position has unlimited capacity and eventually any student is put on the waiting list of a real position $x \in X$ or the null position. Once the algorithm ends, positions are assigned to the students on the respective position waiting lists and the resulting allocation is the student-optimal stable allocation $\varphi^{\succ}(N, R, q)$ for the placement problem $(N, R, q)$.

By $\varphi^{\succ}$ we denote the student-optimal stable mechanism that assigns to each placement problem $(N, R, q)$ the student-optimal stable allocation $\varphi^{\succ}(N, R, q)$.

\section{Consistency}

\subsection{Local Consistency}

Ergin (2002) refers to the student-optimal stable mechanism $\varphi^{\succ}$ as the "best rule" and analyzes for which priority structures $\succ, \varphi^{\succ}$ satisfies well-known and desirable properties given a fixed set of students $N \subseteq \bar{N}$ and a fixed quota vector $q$. Ergin (2002, Theorem 1) provides a necessary and sufficient acyclicity condition (Definition 2 below) for the student-optimal stable mechanism to satisfy either of Pareto efficiency, ${ }^{6}$ group strategy-proofness, ${ }^{7}$ and a (local!) consistency property (Ergin, 2002, p. 2494) that we explain next.

Loosely speaking, a placement mechanism is consistent if, whenever some students leave with their allotments, the placement mechanism allocates the remaining positions among the students who did not leave in the same way as in the original placement problem. In order to introduce consistency of a placement mechanism in a model where the set of agents and resources are fixed a priori, Ergin (2002) only requires a local consistency check for all reduced placement problems that are obtained from an original placement problem $(N, R, q)(N$ and $q$ being fixed $){ }^{8}$ Formally, Ergin (2002, p. 2493) only requires that $\varphi^{\succ}$ is consistent on the domain of reduced placement problems that are obtained from a placement problem $(N, R, q)$ when a subset of agents $N^{\prime} \subseteq N$ reallocates resources after agents in $N \backslash N^{\prime}$ have left with their allotments at $\varphi^{\succ}(N, R, q)$.

\section{Definition 1. Local consistency}

Let $N \subseteq \bar{N}$ be a set of students and $q$ a quota vector. A placement mechanism $\varphi$ is locally consistent for $(N, q)$ if for each profile $R \in \mathcal{R}^{N}$ and each subset of students $N^{\prime} \subseteq N$ : [for all $\left.i \in N^{\prime}, \varphi_{i}\left(N^{\prime}, R_{N^{\prime}}, q\left(N^{\prime}, \varphi(N, R, q)\right)\right)=\varphi_{i}(N, R, q)\right]$.

The reason why Ergin's consistency property takes a "local form" is that the other properties in Ergin's (2002) Theorem 1, Pareto efficiency and group strategy-proof, are local properties as

\footnotetext{
${ }^{6}$ A placement mechanism is Pareto efficient if no assigned allocation can be (Pareto) improved such that all students are weakly better off and some are strictly better off.

${ }^{7}$ A placement mechanism is group strategy-proof if no group of students, by jointly misrepresenting their preferences, can change their allotments such that all members of the group are weakly better off and some are strictly better off.

${ }^{8}$ Thomson and Zhou (1993) take a similar "local consistency" approach in a model with atomless economies.
} 
well, i.e., they are defined for fixed $N$ and $q$. However, for real life placement problems it also might just be more practical and normatively sufficient to require consistency only within each application round (which means that the set of agents and the quota are indeed fixed) and not across all previous, future, and potentially possible application rounds.

Next, we introduce Ergin's (2002, p. 2492) acyclicity condition for priority structures. Again, since the set of agents $N$ and the quota vector $q$ are fixed, acyclicity has a "local character."

Definition 2 (Ergin, 2002). Local cycles and local acyclicity

Let $N \subseteq \bar{N}$ be a set of students and $q$ a quota vector. Given a priority structure $\succ$, a local cycle for $(N, q)$ is constituted of ordered and distinct $x, y \in X\left(q_{x}, q_{y} \neq 0\right)$ and $i, j, k \in N$ such that the following two conditions are satisfied:

cycle condition $i \succ_{x} j \succ_{x} k \succ_{y} i$ and

c-scarcity condition there exist disjoint (and possibly empty) sets $N_{x}, N_{y} \subseteq N \backslash\{i, j, k\}$ such that $N_{x} \subseteq\left\{l \in N: l \succ_{x} j\right\}, N_{y} \subseteq\left\{l \in N: l \succ_{y} i\right\},\left|N_{x}\right|=q_{x}-1$, and $\left|N_{y}\right|=q_{y}-1$.

A priority structure $\succ$ is locally acyclic for $(N, q)$ if it has no local cycles for $(N, q)$.

If quotas are all equal to 1 , then the cycle condition is sufficient to establish the existence of a local cycle. For other quotas, the c-scarcity condition limits the definition of a local cycle to cases where there indeed exist students' preferences such that students $i, j$, and $k$ compete for position types $x$ and $y$ (in the absence of this competition, e.g., because the quotas do in fact not limit the access of the students to positions $x$ and $y$, a local cycle will not lead to the violation of Pareto efficiency, group strategy-proofness, or local consistency - see Ergin, 2002, for further discussion).

Ergin (2002, Theorem 1, (iii) $\Leftrightarrow($ iv), p. 2494) characterizes local consistency of the studentoptimal stable mechanism by local acyclicity (Ergin, 2002, uses the terms consistency and acyclicity without referring to their local character).

Theorem 1 (Ergin, 2002). Local consistency of the student-optimal stable mechanism Let $N \subseteq \bar{N}$ be a set of agents, q a quota vector, and $\succ$ a priority structure. Then, $\varphi^{\succ}$ is locally consistent for $(N, q)$ if and only if $\succ$ is locally acyclic for $(N, q)$.

\subsection{Global Consistency}

In the literature, consistency is usually defined for models with a variable population and variable resources. ${ }^{9}$ In order to distinguish this standard notion of consistency from Ergin's local consistency property, we will refer to it as global consistency.

In our variable population and variable resources extension of Ergin's (2002) model, a mechanism $\varphi$ is globally consistent if for any set of present agents $N$ and for any set of available resources (represented by a quota vector $q$ ), it is locally consistent.

\section{Definition 3. Global consistency}

A placement mechanism $\varphi$ is globally consistent if it is locally consistent for all $(N, q)$ such that $N \subseteq \bar{N}$ and $q$ is a quota vector.

\footnotetext{
${ }^{9}$ See Ergin $(2000)$ and Thomson $(2009,2011)$ for the indivisible-object assignment setting and general allocation problems, respectively.
} 
Using Ergin's (2002) result (Theorem 1), we now identify a necessary and sufficient condition for priority structure $\succ$ to guarantee that $\varphi^{\succ}$ is globally consistent.

\section{Definition 4. Unit cycles and unit acyclicity}

Given a priority structure $\succ$, a unit cycle is constituted of ordered and distinct $x, y \in X$ and $i, j, k \in \bar{N}$ such that the following condition is satisfied:

cycle condition $i \succ_{x} j \succ_{x} k \succ_{y} i$.

A priority structure $\succ$ is unit acyclic if it has no unit cycles.

Corollary 1. Global consistency of the student-optimal stable mechanism Let $\succ$ be a priority structure. Then, $\varphi^{\succ}$ is globally consistent if and only if $\succ$ is unit acyclic.

Proof. Let $\succ$ be a priority structure. By definition, $\varphi^{\succ}$ is globally consistent if for all $N \subseteq \bar{N}$ and all quota vectors $q, \varphi^{\succ}$ is locally consistent for $(N, q)$. By Theorem 1 (Ergin, 2002, Theorem 1, (iii) $\Leftrightarrow($ iv)), this is equivalent to the priority structure $\succ$ being locally acyclic for all $N \subseteq \bar{N}$ and for all quota vectors $q$. We complete the proof by showing that $\succ$ being locally acyclic for all $N \subseteq \bar{N}$ and for all $q$ is equivalent to $\succ$ being unit acyclic.

If $\succ$ is unit acyclic, then the cycle condition in Definition 2 cannot be satisfied for any three distinct agents $i, j, k \in \bar{N}$. Hence, $\succ$ is locally acyclic for all $N \subseteq \bar{N}$ and for all $q$.

Now assume that $\succ$ is not unit acyclic. Hence, there exist distinct $x, y \in X$ and $i, j, k \in \bar{N}$ such that $i \succ_{x} j \succ_{x} k \succ_{y} i$. Let $N=\{i, j, k\}$ and $q$ such that $q_{x}=1, q_{y}=1$, and for all $z \in X \backslash\{x, y\}, q_{z}=0$. Then, we have constructed a local cycle for $(N, q)$.

\section{Converse Consistency}

We are also interested in a property that is closely related to consistency: converse consistency. Converse consistency refers to an inverse of the reduction operation that consistency uses. Given some problem, it requires that if a mechanism or correspondence (partially) chooses an allocation for each of its associated reduced two-agent problems, then the (whole) allocation should be chosen for the problem involving the whole group. Sasaki and Toda (1992) and ÖzkalSanver (2013) consider converse consistency for the closely related class of marriage problems (one-to-one matching problems). Furthermore, Thomson $(2009,2011)$ provides an extensive survey of consistency and its converse for various economic models. Since we focus on the student-optimal stable mechanism, we introduce converse consistency directly for mechanisms and not (as is the standard) for correspondences.

\subsection{Local Converse Consistency}

Similarly as in Section 3, we first introduce a "local version" of converse consistency. For real life placement problems that would mean that converse consistency is only required within an application round but not across different application rounds.

\section{Definition 5. Local converse consistency}

Let $N \subseteq \bar{N}$ be a set of students and $q$ a quota vector. A placement mechanism $\varphi$ is locally 
conversely consistent for $(N, q)$ if for each profile $R \in \mathcal{R}^{N}$ and for each allocation $\alpha$ for placement problem $(N, R, q)$ : [if for all $N^{\prime} \subseteq N$ with $\left|N^{\prime}\right|=2, \varphi\left(N^{\prime}, R_{N^{\prime}}, q\left(N^{\prime}, \alpha\right)\right)=\alpha_{N^{\prime}}$, then $\varphi(N, R, q)=\alpha]$.

The following example demonstrates that a student-optimal stable mechanism might violate local converse consistency (our example is a simplification of Özkal-Sanver's, 2013, Example 2.1).

Example 1. A priority structure $\succ$ such that $\varphi^{\succ}$ is not locally conversely consistent Let $X=\{x, y, z\}, N=\{1,2,3\}$, and $q_{x}=q_{y}=q_{z}=1$. Consider the priority structure $\succ$ and preferences $R \in \mathcal{R}^{N}$ as given in the tables below.

\begin{tabular}{|c|c|c|}
\hline$\succ_{x}$ & $\succ_{y}$ & $\succ_{z}$ \\
\hline \hline 1 & 2 & 3 \\
2 & 3 & 1 \\
3 & 1 & 2 \\
\hline
\end{tabular}

and

\begin{tabular}{|c|c|c|}
\hline$R_{1}$ & $R_{2}$ & $R_{3}$ \\
\hline \hline$z$ & $x$ & $y$ \\
$x$ & $y$ & $z$ \\
\hline
\end{tabular}

For this placement problem, $\varphi^{\succ}(N, R, q)=(z, x, y)$. Let $\alpha=(x, y, z)$. One easily verifies that for all $N^{\prime} \subseteq N$ with $\left|N^{\prime}\right|=2, \varphi^{\succ}\left(N^{\prime}, R_{N^{\prime}}, q\left(N^{\prime}, \alpha\right)\right)=\alpha_{N^{\prime}}$. However, $\varphi^{\succ}(N, R, q) \neq \alpha$. Therefore, $\varphi^{\succ}$ is not locally conversely consistent for $(N, q)$. (Incidentally, notice that $\alpha$ is the position-type-optimal stable matching of the associated marriage problem.) Observe that $1 \succ_{x} 2 \succ_{y} 3 \succ_{z} 1$, which turns out to be the "problematic part" in priority structure $\succ$ that causes $\varphi^{\succ}$ to violate local converse consistency.

Given a fixed set of students $N$ and a fixed quota vector $q$, we first analyze which priority structures $\succ$ guarantee that the student-optimal stable mechanism $\varphi^{\succ}$ is locally conversely consistent for $(N, q)$. In line with Ergin's (2002) result, we show that local acyclicity of the priority structure for $(N, q)$ is sufficient for $\varphi^{\succ}$ to be locally conversely consistent for $(N, q)$. However, it turns out that the class of priority structures that induce $\varphi^{\succ}$ to be locally conversely consistent for $(N, q)$ is strictly larger than the class of locally acyclic priority structures for $(N, q)$.

We first introduce local shift-freeness of a priority structure $\succ$ for $(N, q)$. We then prove that local shift-freeness for $(N, q)$ is a necessary and sufficient condition for the student-optimal stable mechanism $\varphi^{\succ}$ to be locally conversely consistent for $(N, q)$ (Theorem 2).

\section{Definition 6. Local shifts and local shift-freeness}

Let $N \subseteq \bar{N}$ be a set of students and $q$ a quota vector. Given a priority structure $\succ$, a local shift for $(N, q)$ is constituted of ordered and distinct $x, y, z \in X\left(q_{x}, q_{y}, q_{z} \neq 0\right)$ and $i, j, k \in N$ such that the following two conditions are satisfied:

shift condition $i \succ_{x} j \succ_{y} k \succ_{z} i$ and

s-scarcity condition there exist disjoint (and possibly empty) sets $N_{x}, N_{y}, N_{z} \subseteq N \backslash\{i, j, k\}$ such that $N_{x} \subseteq\left\{l \in N: l \succ_{x} j\right\}, N_{y} \subseteq\left\{l \in N: l \succ_{y} k\right\}, N_{z} \subseteq\left\{l \in N: l \succ_{z} i\right\},\left|N_{x}\right|=q_{x}-1$, $\left|N_{y}\right|=q_{y}-1$, and $\left|N_{z}\right|=q_{z}-1$.

A priority structure $\succ$ is locally shift-free for $(N, q)$ if it has no local shifts for $(N, q)$. 
If quotas are all equal to 1 , then the shift condition is sufficient to establish the existence of a local shift. For other quotas, the s-scarcity condition limits the definition of a local shift to cases where there indeed exist students' preferences such that students $i, j$, and $k$ compete for position types $x, y$, and $z$ (in the absence of this competition, e.g., because the quotas do in fact not limit the access of the students to positions $x, y$, and $z$, a local shift will not cause the student-optimal stable mechanism $\varphi^{\succ}$ to violate local converse consistency).

Note that Example 1 exhibits a local " 3 -shift" in the sense that it involves 3 students (and 3 position types). We will use the following concept of more general local shifts to prove our main result.

\section{Definition 7. Local $k$-shifts}

Let $N \subseteq \bar{N}$ be a set of students and $q$ a quota vector. Given a priority structure $\succ$, a local $k$-shift for $(N, q)$ is constituted of ordered and distinct $x_{1}, \ldots, x_{k} \in X\left(q_{x_{1}}, \ldots, q_{x_{k}} \neq 0\right)$ and $i_{1}, \ldots, i_{k} \in N$ with $k \geq 3$ such that the following two conditions are satisfied:

$k$-shift condition $i_{1} \succ_{x_{1}} i_{2} \succ_{x_{2}} i_{3} \succ_{x_{3}} \cdots \succ_{x_{k-1}} i_{k} \succ_{x_{k}} i_{k+1}:=i_{1}$ and

s-scarcity condition there exist disjoint (and possibly empty) sets $N_{x_{l}} \subseteq N \backslash\left\{i_{1}, \ldots, i_{k}\right\}(l=$ $1, \ldots, k)$ such that $N_{x_{l}} \subseteq\left\{j \in N: j \succ_{x_{l}} i_{l+1}\right\}$ and $\left|N_{x_{l}}\right|=q_{x_{l}}-1(l=1, \ldots, k)$.

We next show that for any pair $(N, q)$, the presence of a local $k$-shift for $(N, q)$ implies the presence of a local shift for $(N, q)$.

Lemma 1. Let $N \subseteq \bar{N}$ be a set of students and $q$ a quota vector. If a priority structure $\succ$ has a local $k$-shift for $(N, q)$, then it has a local shift for $(N, q)$.

Proof. Assume that priority structure $\succ$ has a local $k$-shift for $(N, q)$ with $k>3$. We prove that then it also has a local shift for $(N, q)$ (i.e., a local 3-shift for $(N, q))$.

CASE I: $\succ$ has a local 4 -shift for $(N, q)$.

Assume that $x_{1}, x_{2}, x_{3}, x_{4} \in X\left(q_{x_{1}}, \ldots, q_{x_{4}} \neq 0\right)$ and (without loss of generality) $1,2,3,4 \in N$ constitute a local 4-shift for $(N, q)$ (with the required $N_{x_{1}}, N_{x_{2}}, N_{x_{3}}, N_{x_{4}}$ ) such that (part of) the priority structure restricted to $\left\{x_{1}, x_{2}, x_{3}, x_{4}\right\}$ and $\{1,2,3,4\}$ exhibits the structure given by the next table.

\begin{tabular}{|c|c|c|c|}
\hline$\succ_{x_{1}}$ & $\succ_{x_{2}}$ & $\succ_{x_{3}}$ & $\succ_{x_{4}}$ \\
\hline \hline 1 & 2 & 3 & 4 \\
2 & 3 & 4 & 1 \\
\hline
\end{tabular}

Assume $2 \succ_{x_{1}}$ 3. Then, $x_{1}, x_{3}, x_{4}$ and 1,3,4 constitute a local shift for $(N, q)$ (with $N_{x_{1}}, N_{x_{3}}$, and $N_{x_{4}}$ from the original local 4-shift for $(N, q)$ ). Similarly, $3 \succ_{x_{2}} 4,4 \succ_{x_{3}} 1$, and $1 \succ_{x_{4}} 2$ lead to local shifts for $(N, q)$. Thus, assume that $3 \succ_{x_{1}} 2,4 \succ_{x_{2}} 3,1 \succ_{x_{3}} 4$, and $2 \succ_{x_{4}} 1$. Then, (part of) the priority structure restricted to $\left\{x_{1}, x_{2}, x_{3}, x_{4}\right\}$ and $\{1,2,3,4\}$ exhibits the structure given by the next table where the notation $[i, j]$ for position type $x$ means that either $i \succ_{x} j$ or $j \succ_{x} i$.

\begin{tabular}{|c|c|c|c|}
\hline$\succ_{x_{1}}$ & $\succ_{x_{2}}$ & $\succ_{x_{3}}$ & $\succ_{x_{4}}$ \\
\hline \hline$[1,3]$ & {$[2,4]$} & {$[1,3]$} & {$[2,4]$} \\
2 & 3 & 4 & 1 \\
\hline
\end{tabular}


Assume $4 \succ_{x_{1}}$ 2. Then $x_{4}, x_{3}, x_{1}$ and 2,1,4 constitute a local shift for $(N, q)$ (with $N_{x_{4}}, N_{x_{3}}$, and $N_{x_{1}}$ from the original local 4-shift for $(N, q)$ ). Similarly, $1 \succ_{x_{2}} 3,2 \succ_{x_{3}} 4$, and $3 \succ_{x_{4}} 1$ lead to local shifts for $(N, q)$. Thus, assume that $2 \succ_{x_{1}} 4,3 \succ_{x_{2}} 1,4 \succ_{x_{3}} 2$, and $1 \succ_{x_{4}} 3$. Then, (part of) the priority structure restricted to $\left\{x_{1}, x_{2}, x_{3}, x_{4}\right\}$ and $\{1,2,3,4\}$ exhibits the structure given by the next table.

\begin{tabular}{|c|c|c|c|}
\hline$\succ_{x_{1}}$ & $\succ_{x_{2}}$ & $\succ_{x_{3}}$ & $\succ_{x_{4}}$ \\
\hline \hline$[1,3]$ & {$[2,4]$} & {$[1,3]$} & {$[2,4]$} \\
2 & 3 & 4 & 1 \\
4 & 1 & 2 & 3 \\
\hline
\end{tabular}

Then, $x_{1}, x_{2}, x_{4}$ and $3,4,1$ constitute a local shift for $(N, q)$ (with $N_{x_{1}}, N_{x_{2}}$, and $N_{x_{4}}$ from the original local 4 -shift for $(N, q))$.

CASE II: $\succ$ has a local $k$-shift for $(N, q)$ with $k \geq 5$.

Assume that $x_{1}, \ldots, x_{k} \in X\left(q_{x_{1}}, \ldots, q_{x_{k}} \neq 0\right)$ and (without loss of generality) $1, \ldots, k \in N$ constitute a local $k$-shift for $(N, q)$ (with the required $N_{x_{1}}, \ldots, N_{x_{k}}$ ) such that (part of) the priority structure restricted to $\left\{x_{1}, \ldots, x_{k}\right\}$ and $\{1, \ldots, k\}$ exhibits the structure given by the next table.

\begin{tabular}{|c|c|c|c|c|c|c|}
\hline$\succ_{x_{1}}$ & $\succ_{x_{2}}$ & $\succ_{x_{3}}$ & $\succ_{x_{4}}$ & $\cdots$ & $\succ_{x_{k-1}}$ & $\succ_{x_{k}}$ \\
\hline \hline 1 & 2 & 3 & 4 & $\cdots$ & $k-1$ & $k$ \\
2 & 3 & 4 & 5 & $\cdots$ & $k$ & $k+1:=1$ \\
\hline
\end{tabular}

Assume $1 \succ_{x_{k}}$ 3. Then, $x_{3}, x_{4}, \ldots, x_{k}$ and $3,4, \ldots, k-1, k$ constitute a local $(k-2)$-shift for $(N, q)$ (with $N_{x_{3}}, N_{x_{4}}, \ldots, N_{x_{k}}$ from the original local $k$-shift for $(N, q)$ ). Note that $k-2 \geq 3$. Similarly, for each $l \in\{1, \ldots, k\}, l+1 \succ_{x_{l}} l+3$ leads to a local $(k-2)$-shift for $(N, q)$ (with the convention to use the modulo $k$ operator such that $k+2=2, k+3=3$, etc.). Thus, assume for all $l=1, \ldots, k$ that $l+3 \succ_{x_{l}} l+1$. Then, (part of) the priority structure restricted to $\left\{x_{1}, \ldots, x_{k}\right\}$ and $\{1, \ldots, k\}$ exhibits the structure given by the next table.

\begin{tabular}{|c|c|c|c|c|c|c|}
\hline$\succ_{x_{1}}$ & $\succ_{x_{2}}$ & $\succ_{x_{3}}$ & $\succ_{x_{4}}$ & $\cdots$ & $\succ_{x_{k-1}}$ & $\succ_{x_{k}}$ \\
\hline \hline$[1,4]$ & {$[2,5]$} & {$[3,6]$} & {$[4,7]$} & $\cdots$ & {$[k-1,2]$} & {$[k, 3]$} \\
2 & 3 & 4 & 5 & $\cdots$ & $k$ & $k+1:=1$ \\
\hline
\end{tabular}

Then, $x_{2}, x_{3}, x_{4}$ and 5,3,4 constitute a local 3-shift for $(N, q)$ (with $N_{x_{2}}, N_{x_{3}}$, and $N_{x_{4}}$ from the original local $k$-shift for $(N, q))$. To summarize Case II, if $\succ$ has a local $k$-shift for $(N, q)$ with $k \geq 5$ then it has also a local $(k-2)$-shift for $(N, q)$ or a local 3-shift for $(N, q)$. In case of a local $(k-2)$-shift for $(N, q)$, depending on $(k-2)=4$ or $(k-2) \geq 5$, we repeat the arguments in Case I or II, and find a local 3 -shift for $(N, q)$ in finitely many steps.

We are now ready to state our main result for local converse consistency.

Theorem 2. Local converse consistency of the student-optimal stable mechanism Let $N \subseteq \bar{N}$ be a set of agents, q a quota vector, and $\succ$ a priority structure. Then, $\varphi^{\succ}$ is locally conversely consistent for $(N, q)$ if and only if $\succ$ is locally shift-free for $(N, q)$. 
Proof.

PART I: Assume that priority structure $\succ$ has a local shift for $(N, q)$. We show that then $\varphi^{\succ}$ is not locally conversely consistent for $(N, q)$.

Assume that $x, y, z \in X\left(q_{x}, q_{y}, q_{z} \neq 0\right)$ and (without loss of generality) $1,2,3 \in N$ constitute a local shift for $(N, q)$ (with the required $\left.N_{x}, N_{y}, N_{z}\right)$ such that (part of) the priority structure restricted to $\{x, y, z\}$ and $\{1,2,3\}$ exhibits the structure given by the next table.

\begin{tabular}{|c|c|c|}
\hline$\succ_{x}$ & $\succ_{y}$ & $\succ_{z}$ \\
\hline \hline 1 & 2 & 3 \\
2 & 3 & 1 \\
\hline
\end{tabular}

Let $\tilde{N}=\{1,2,3\} \cup N_{x} \cup N_{y} \cup N_{z}$ and $R \in \mathcal{R}^{N}$ be such that

\begin{tabular}{|c|c|c|c|c|c|c|}
\hline$R_{1}$ & $R_{2}$ & $R_{3}$ & $R_{i}\left(i \in N_{x}\right)$ & $R_{i}\left(i \in N_{y}\right)$ & $R_{i}\left(i \in N_{z}\right)$ & $R_{i}(i \in N \backslash \tilde{N})$ \\
\hline \hline$z$ & $x$ & $y$ & $x$ & $y$ & $z$ & 0 \\
$x$ & $y$ & $z$ & & & & \\
\hline
\end{tabular}

and any position types that are not depicted in the preference table are unacceptable. For placement problem $(N, R, q)$, the student-optimal stable mechanism assigns to all students their preferred position, i.e., $\varphi_{i}^{\succ}(N, R, q)=x\left(i \in N_{x} \cup\{2\}\right), \varphi_{i}^{\succ}(N, R, q)=y\left(i \in N_{y} \cup\{3\}\right)$, $\varphi_{i}^{\succ}(N, R, q)=z\left(i \in N_{z} \cup\{1\}\right)$, and $\varphi_{i}^{\succ}(N, R, q)=0(i \in N \backslash \tilde{N})$.

Now, consider allocation $\alpha$ for placement problem $(N, R, q)$ that is obtained from $\varphi_{i}^{\succ}(N, R, q)$ by giving agents 1,2 , and 3 their second choice position and not changing the allotments of the remaining agents, i.e., $\alpha_{i}=x\left(i \in N_{x} \cup\{1\}\right), \alpha_{i}=y\left(i \in N_{y} \cup\{2\}\right), \alpha_{i}=z\left(i \in N_{z} \cup\{3\}\right)$, and $\alpha_{i}=0(i \in N \backslash \tilde{N})$.

Note that for placement problem $(N, R, q)$, both allocations $\varphi^{\succ}(N, R, q)$ and $\alpha$ allocate all available positions of position types $x, y$, and $z$. Moreover, all other position types are unacceptable for all students in $N$.

We prove that for all $N^{\prime} \subseteq N$ with $\left|N^{\prime}\right|=2, \varphi^{\succ}\left(N^{\prime}, R_{N^{\prime}}, q\left(N^{\prime}, \alpha\right)\right)=\alpha_{N^{\prime}}$. If $N^{\prime} \cap(N \backslash \tilde{N}) \neq \emptyset$, then immediately $\varphi^{\succ}\left(N^{\prime}, R_{N^{\prime}}, q\left(N^{\prime}, \alpha\right)\right)=\alpha_{N^{\prime}}$. Assume $N^{\prime} \cap(N \backslash \tilde{N})=\emptyset$.

CASE I: $N^{\prime} \cap\{1,2,3\} \neq \emptyset$. Let $N^{\prime}=\{i, j\}$. The table below summarizes the relevant information to easily check that $\varphi^{\succ}\left(N^{\prime}, R_{N^{\prime}}, q\left(N^{\prime}, \alpha\right)\right)=\alpha_{N^{\prime}}$ (note that for all other choices of $j$, both agents $i$ and $j$ receive their best available position).

\begin{tabular}{|c|c|c|c|c|}
\hline$i=$ & $j \in$ & $\left(\alpha_{i}, \alpha_{j}\right)$ & both prefer & decisive priority \\
\hline \hline 1 & $N_{z} \cup\{3\}$ & $(x, z)$ & $z$ & $j \succ_{z} i$ \\
2 & $N_{x} \cup\{1\}$ & $(y, x)$ & $x$ & $j \succ_{x} i$ \\
3 & $N_{y} \cup\{2\}$ & $(z, y)$ & $y$ & $j \succ_{y} i$ \\
\hline
\end{tabular}

CASE II: $N^{\prime} \cap\{1,2,3\}=\emptyset$. Let $N^{\prime}=\{i, j\}$. Since $i, j \in N_{x} \cup N_{y} \cup N_{z}$, students $i$ and $j$ obtain the same (and their best) position at both allocations $\varphi^{\succ}\left(N^{\prime}, R_{N^{\prime}}, q\left(N^{\prime}, \alpha\right)\right)$ and $\alpha$. Hence, $\varphi^{\succ}\left(N^{\prime}, R_{N^{\prime}}, q\left(N^{\prime}, \alpha\right)\right)=\alpha_{N^{\prime}}$. 
If $\varphi^{\succ}$ was locally conversely consistent for $(N, q)$, then $\varphi^{\succ}(N, R, q)=\alpha$, which is clearly not the case.

PART II: Next, assume that $\varphi^{\succ}$ is not locally conversely consistent for $(N, q)$. We show that then the priority structure $\succ$ has a local shift for $(N, q)$.

By Lemma 1, it is sufficient to show that the priority structure has a local $k$-shift for $(N, q)$ for some $k \geq 3$.

Since $\varphi^{\succ}$ is not locally conversely consistent for $(N, q)$, there exist preferences $R \in \mathcal{R}^{N}$ and an allocation $\alpha$ for $(N, R, q)$ such that $\varphi^{\succ}(N, R, q) \neq \alpha$ and for all $N^{\prime} \subseteq N$ with $\left|N^{\prime}\right|=2$, $\varphi^{\succ}\left(N^{\prime}, R_{N^{\prime}}, q\left(N^{\prime}, \alpha\right)\right)=\alpha_{N^{\prime}}$.

Since for all $N^{\prime} \subseteq N$ with $\left|N^{\prime}\right|=2, \varphi^{\succ}\left(N^{\prime}, R_{N^{\prime}}, q\left(N^{\prime}, \alpha\right)\right)=\alpha_{N^{\prime}}$ it follows from the definition of $\varphi^{\succ}$ that $\alpha$ is individually rational. Suppose allocation $\alpha$ violates the priority of a student $i \in N$ for placement problem $(N, R, q)$. Then, there exists a position type $x \in X$ and a student $j \in N \backslash\{i\}$ such that $i \succ_{x} j, \alpha_{j}=x$, and $x P_{i} \alpha_{i}$. Let $N^{\prime}=\{i, j\}$. Then, the reduced allocation $\alpha_{N^{\prime}}$ also violates the priority of $i \in N$ for placement problem $\left(N^{\prime}, R_{N^{\prime}}, q\left(N^{\prime}, \alpha\right)\right)$. However, since $\varphi^{\succ}\left(N^{\prime}, R_{N^{\prime}}, q\left(N^{\prime}, \alpha\right)\right)=\alpha_{N^{\prime}}$ and $\varphi^{\succ}$ is a fair mechanism, such a violation of priority is impossible. Hence, allocation $\alpha$ does not violate the priority of any student in $N$ for placement problem $(N, R, q)$. A similar argument shows that allocation $\alpha$ is non-wasteful for placement problem $(N, R, q)$.

Since for placement problem $(N, R, q), \alpha$ is individually rational, non-wasteful, and it does not violate the priority of any student, allocation $\alpha$ corresponds to a stable matching in the associated college admissions problem. Hence, it follows from Roth $\left(1985\right.$, Theorem $\left.2^{*}\right)$ that for all $i \in N, \varphi_{i}^{\succ}(N, R, q) R_{i} \alpha_{i}$. From Roth (1984, Theorem 9) it follows that for all $x \in X, \mid\{i \in N$ : $\left.\varphi_{i}^{\succ}(N, R, q)=x\right\}|=|\left\{i \in N: \alpha_{i}=x\right\} \mid$. Therefore, for each $i \in N$ with $\varphi_{i}^{\succ}(N, R, q) \neq \alpha_{i}$ (i.e., $\left.\varphi_{i}^{\succ}(N, R, q) P_{i} \alpha_{i}\right)$ there exists $j \in N \backslash\{i\}$ with $\alpha_{j}=\varphi_{i}^{\succ}(N, R, q)$ and $\varphi_{j}^{\succ}(N, R, q) \neq \alpha_{j}$. Since $\varphi^{\succ}(N, R, q) \neq \alpha$ and $|N|$ is finite, the previous observation establishes the existence of a group of students (without loss of generality) $\{1, \ldots, k\}$ such that $\varphi_{1}^{\succ}(N, R, q) P_{1} \alpha_{1}=\varphi_{2}^{\succ}(N, R, q) P_{2} \alpha_{2}=$ $\ldots=\varphi_{k}^{\succ}(N, R, q) P_{k} \alpha_{k}=\varphi_{1}^{\succ}(N, R, q)$.

Assume $k=2$. Then, $\alpha_{2} P_{1} \alpha_{1} P_{2} \alpha_{2}$. By assumption that for all $N^{\prime} \subseteq N$ with $\left|N^{\prime}\right|=2$, $\varphi^{\succ}\left(N^{\prime}, R_{N^{\prime}}, q\left(N^{\prime}, \alpha\right)\right)=\alpha_{N^{\prime}}$, it follows that $\varphi^{\succ}\left(\{1,2\}, R_{\{1,2\}}, q(\{1,2\}, \alpha)\right)=\left(\alpha_{1}, \alpha_{2}\right)$. Thus, $\left(\alpha_{2}, \alpha_{1}\right)$ strictly Pareto dominates $\varphi^{\succ}\left(\{1,2\}, R_{\{1,2\}}, q(\{1,2\}, \alpha)\right)$ in the college admissions market associated with $\left(\{1,2\}, R_{\{1,2\}}, q(\{1,2\}, \alpha)\right)$; contradicting Roth (1982, Theorem 6). ${ }^{10}$ Hence, $k>2$.

From Roth and Sotomayor (1990, Theorem 5.27), by the polarization of interests between the two sides of the associated college admissions market at the two stable (associated) matchings $\varphi^{\succ}(N, R, q)$ and $\alpha$, we have $1 \succ_{\alpha_{1}} 2 \succ_{\alpha_{2}} 3 \succ_{\alpha_{3}} \cdots \succ_{\alpha_{k-1}} k \succ_{\alpha_{k}} 1$. So, (part of) the priority structure restricted to $\left\{\alpha_{1}, \ldots, \alpha_{k}\right\}$ and $\{1, \ldots, k\}$ exhibits the structure given by the next table.

\begin{tabular}{|c|c|c|c|c|c|c|}
\hline$\succ_{\alpha_{1}}$ & $\succ_{\alpha_{2}}$ & $\succ_{\alpha_{3}}$ & $\succ_{\alpha_{4}}$ & $\cdots$ & $\succ_{\alpha_{k-1}}$ & $\succ_{\alpha_{k}}$ \\
\hline \hline 1 & 2 & 3 & 4 & $\cdots$ & $k-1$ & $k$ \\
2 & 3 & 4 & 5 & $\cdots$ & $k$ & $k+1:=1$ \\
\hline
\end{tabular}

\footnotetext{
${ }^{10}$ Because economy $\left(\{1,2\}, R_{\{1,2\}}, q(\{1,2\}, \alpha)\right)$ might contain additional positions apart from $\alpha_{1}$ and $\alpha_{2}$, we cannot immediately obtain a contradiction by directly calculating $\varphi^{\succ}\left(\{1,2\}, R_{\{1,2\}}, q(\{1,2\}, \alpha)\right)=\left(\alpha_{2}, \alpha_{1}\right)($ an argument along these lines would involve non-wastefulness for the original economy $(N, R, q)$ ).
} 
Note that so far we have found distinct $\alpha_{1}, \ldots, \alpha_{k} \in X\left(q_{\alpha_{1}}, \ldots, q_{\alpha_{k}} \neq 0\right)$ and $1, \ldots, k \in N$ with $k \geq 3$ that satisfy the $k$-shift condition in Definition 7 . We now show that also the s-scarcity condition is satisfied.

Let $l \in\{1, \ldots, k\}$. Let $i \in N$ such that $\alpha_{i}=\alpha_{l}$. Let $N^{*}=\{i, l+1\}$. The only two positions assigned at $\alpha$ to agents in $N^{*}$ for placement problem $(N, R, q)$ are $\alpha_{l}$ and $\alpha_{l+1}$. By our choice of $\alpha$ (in violation of local converse consistency for $(N, q)$ ), $\alpha_{l}$ and $\alpha_{l+1}$ then are the only two positions assigned at $\varphi^{\succ}\left(N^{*}, R_{N^{*}}, q\left(N^{*}, \alpha\right)\right)$. Recall that $\alpha_{l} P_{l+1} \alpha_{l+1}$. Therefore, if $l+1 \succ_{\alpha_{l}} i$, then $\varphi_{l+1}^{\succ}\left(N^{*}, R_{N^{*}}, q\left(N^{*}, \alpha\right)\right)=\alpha_{l} \neq \alpha_{l+1}$; contradicting that $\alpha_{N^{*}}=\varphi^{\succ}\left(N^{*}, R_{N^{*}}, q\left(N^{*}, \alpha\right)\right)$. Hence, for all $l \in\{1, \ldots, k\}$ and for all $i \in N$ with $\alpha_{i}=\alpha_{l}, i \succ_{\alpha_{l}} l+1$. Thus, for all $l \in\{1, \ldots, k\}$, $N_{\alpha_{l}}:=\left\{i \in N: \alpha_{i}=\alpha_{l}\right\} \backslash\{l\}$ satisfies $N_{\alpha_{l}} \subseteq\left\{i \in N: i \succ_{\alpha_{l}} l+1\right\}$. Moreover, since for all $l \in\{1, \ldots, k\}, \alpha_{l} P_{l+1} \alpha_{l+1}$, it follows from non-wastefulness of $\alpha$ that $\left|N_{\alpha_{l}}\right|=q_{\alpha_{l}}-1$. Finally, the sets $N_{\alpha_{1}}, \ldots, N_{\alpha_{k}}$ are mutually disjoint by definition. Hence, $\alpha_{1}, \ldots, \alpha_{k}$ and $1, \ldots, k$ constitute a local $k$-shift for $(N, q)$ (with the required $N_{\alpha_{1}}, \ldots, N_{\alpha_{k}}$ ).

Next, we show that local acyclicity of a priority structure $\succ$ for $(N, q)$ implies that it is locally shift-free for $(N, q)$ as well (Lemma 2), and therefore, the local acyclicity of priority structure $\succ$ for $(N, q)$ implies that the student-optimal stable mechanism $\varphi^{\succ}$ is locally conversely consistent for $(N, q)$ (Corollary 2).

\section{Lemma 2. Local acyclicity implies local shift-freeness}

Let $N \subseteq \bar{N}$ be a set of students and $q$ a quota vector. If a priority structure $\succ$ is locally acyclic for $(N, q)$, then it is locally shift-free for $(N, q)$. Moreover, local acyclicity for $(N, q)$ is in general not equivalent to local shift-freeness for $(N, q)$.

Proof.

PART I: Assume that $x, y, z \in X\left(q_{x}, q_{y}, q_{z} \neq 0\right)$ and $1,2,3 \in N$ constitute a local shift for $(N, q)$ (with the required $N_{x}, N_{y}, N_{z}$ ). So, (part of) the priority structure restricted to $\{x, y, z\}$ and $\{1,2,3\}$ exhibits the structure given by the next table.

\begin{tabular}{|c|c|c|}
\hline$\succ_{x}$ & $\succ_{y}$ & $\succ_{z}$ \\
\hline \hline 1 & 2 & 3 \\
2 & 3 & 1 \\
\hline
\end{tabular}

If $2 \succ_{x} 3$, then $x, z$ and $1,2,3$ constitute a local cycle for $(N, q)$ (with $N_{x}$ and $N_{y}$ from the original shift for $q$ ). Similarly, $3 \succ_{y} 1$ and $1 \succ_{z} 2$ lead to local cycles for $(N, q)$. Thus, assume $3 \succ_{x} 2,1 \succ_{y} 3$, and $2 \succ_{z} 1$. Then, (part of) the priority structure restricted to $\{x, y, z\}$ and $\{1,2,3\}$ exhibits the structure given by the next table where the notation $[i, j]$ for position type $x$ means that either $i \succ_{x} j$ or $j \succ_{x} i$.

\begin{tabular}{|c|c|c|}
\hline$\succ_{x}$ & $\succ_{y}$ & $\succ_{z}$ \\
\hline \hline$[1,3]$ & {$[1,2]$} & {$[2,3]$} \\
2 & 3 & 1 \\
\hline
\end{tabular}

CASE I: $3 \succ_{x} 1$. Then, $3 \succ_{x} 1 \succ_{x} 2$. Assume $q_{x}>1$ and there is $l \in N_{x}$ with $1 \succ_{x} l \succ_{x} 2$. Let $N_{x}^{\prime}=\left(N_{x} \backslash\{l\}\right) \cup\{3\}$. Then, $x, z$ and $1, l, 2$ constitute a local cycle for $(N, q)$ (with $N_{x}^{\prime}$ 
and $N_{z}$ from the original local shift for $\left.(N, q)\right)$. Now assume $q_{x}=1$ or there is no $l \in N_{x}$ with $1 \succ_{x} l \succ_{x} 2$. Then, $x, y$ and $3,1,2$ constitute a local cycle for $(N, q)$ (with $N_{x}$ and $N_{z}$ from the original local shift for $(N, q))$.

CASE II: $1 \succ_{x} 3$. Then, $1 \succ_{x} 3 \succ_{x} 2$. Assume $q_{x}>1$ and there is $l \in N_{x}$ with $3 \succ_{x} l \succ_{x} 2$. Let $N_{x}^{\prime}=\left(N_{x} \backslash\{l\}\right) \cup\{1\}$. Then, $x, y$ and $3, l, 2$ constitute a local cycle for $(N, q)$ (with $N_{x}^{\prime}$ and $N_{y}$ from the original local shift for $(N, q)$ ). Now assume $q_{x}=1$ or there is no $l \in N_{x}$ with $3 \succ_{x} l \succ_{x} 2$. Then, $x, z$ and 1,3,2 constitute a local cycle for $(N, q)$ (with $N_{x}$ and $N_{z}$ from the original local shift for $(N, q))$.

PART II: The following student placement problem shows that in general local acyclicity for $(N, q)$ is a strictly stronger condition than local shift-freeness for $(N, q)$. Let $N=\{1,2,3\}$ and $X=\{x, y, z\}$ with $q_{x}=q_{y}=1$ and $q_{z}=2$. The priority structure $\succ$ is given in the next table.

\begin{tabular}{|c|c|c|}
\hline$\succ_{x}$ & $\succ_{y}$ & $\succ_{z}$ \\
\hline \hline 1 & 2 & 3 \\
2 & 3 & 1 \\
3 & 1 & 2 \\
\hline
\end{tabular}

One easily verifies that $x, y$ and $1,2,3$ constitute a local cycle for $(N, q)$, but that there is no local shift for $(N, q)$ (because the s-scarcity condition for $z$ cannot be satisfied).

From Theorems 1, 2, and Lemma 2 we obtain the following corollary.

Corollary 2. Let $N \subseteq \bar{N}$ be a set of students and $q$ a quota vector. If a priority structure $\succ$ is locally acyclic for $(N, q)$, then $\varphi^{\succ}$ is locally conversely consistent for $(N, q)$. Thus, if $\varphi^{\succ}$ is locally consistent for $(N, q)$, then $\varphi^{\succ}$ is also locally conversely consistent for $(N, q)$.

For the special case when there is at most one position per position type, local acyclicity of a priority structure $\succ$ for $(N, q)$ is equivalent to local shift-freeness of $\succ$ for $(N, q)$ (Lemma 3) and local acyclicity of $\succ$ for $(N, q)$ is a necessary and sufficient condition for the student-optimal stable mechanism $\varphi^{\succ}$ to be locally conversely consistent for $(N, q)$ (Corollary 3 ).

We call a quota vector $q$ with $q_{x} \in\{0,1\}(x \in X)$ a unit quota vector.

\section{Lemma 3. Quotas equal to one: local shift-freeness implies local acyclicity}

Let $N \subseteq \bar{N}$ be a set of students and $q$ a unit quota vector. If a priority structure $\succ$ is locally shift-free for $(N, q)$, then it is also locally acyclic for $(N, q)$. Thus, a priority structure $\succ$ is locally shift-free for $(N, q)$ if and only if it is locally acyclic for $(N, q)$.

Proof. First note that for any unit quota vector $q$ the c-scarcity condition and the s-scarcity condition are vacuously satisfied. By Lemma 2 , the presence of a local shift for $(N, q)$ implies the presence of a local cycle for $(N, q)$.

Next, we prove that the presence of a local cycle for $(N, q)$ implies the presence of a local shift for $(N, q)$. Suppose without loss of generality that $x, y \in X\left(q_{x}, q_{y} \neq 0\right)$ and $1,2,3 \in N$ constitute a local cycle for $(N, q)$ such that $1 \succ_{x} 2 \succ_{x} 3 \succ_{y} 1$. Since $|X| \geq 3$, let $z \in X \backslash\{x, y\}$. CASE I: $3 \succ_{y} 2$. If $2 \succ_{z} 1 \succ_{z} 3,2 \succ_{z} 3 \succ_{z} 1$, or $1 \succ_{z} 2 \succ_{z} 3$, then $z, y, x$ together with $2,3,1$ constitute a local shift for $(N, q)$. If $3 \succ_{z} 2 \succ_{z} 1$, then $x, y, z$ together with $1,3,2$ constitute a 
local shift for $(N, q)$. Finally, if $1 \succ_{z} 3 \succ_{z} 2$ or $3 \succ_{z} 1 \succ_{z} 2$ then $x, y, z$ together with $2,3,1$ constitute a local shift for $(N, q)$.

CASE II: $2 \succ_{y} 3 \succ_{y} 1$. If $3 \succ_{z} 1 \succ_{z} 2$ or $3 \succ_{z} 2 \succ_{z} 1$ then $x, y, z$ together with $1,2,3$ constitute a local shift for $(N, q)$. If $1 \succ_{z} 2 \succ_{z} 3$ or $1 \succ_{z} 3 \succ_{z} 2$, then $x, y, z$ together with $2,3,1$ constitute a local shift for $(N, q)$. Finally, if $2 \succ_{z} 1 \succ_{z} 3$ or $2 \succ_{z} 3 \succ_{z} 1$ then $z, y, x$ together with $2,3,1$ constitute a local shift for $(N, q)$.

Corollary 3. Let $N \subseteq \bar{N}$ be a set of students and $q$ a unit quota vector. Then, a priority structure $\succ$ is locally acyclic for $(N, q)$ if and only if $\varphi^{\succ}$ is locally conversely consistent for $(N, q)$. Thus, $\varphi^{\succ}$ is locally consistent for $(N, q)$ if and only if $\varphi^{\succ}$ is locally conversely consistent for $(N, q)$.

\subsection{Global Converse Consistency}

In the literature, also converse consistency is usually defined for models with a variable population and variable resources.

\section{Definition 8. Global converse consistency}

A placement mechanism $\varphi$ is globally conversely consistent if it is locally conversely consistent for all $(N, q)$ such that $N \subseteq \bar{N}$ and $q$ is a quota vector.

We are now ready to state our main result for global converse consistency, which parallels Corollary 1 for global consistency.

Corollary 4. Global converse consistency of the student-optimal stable mechanism Let $\succ$ be a priority structure. Then, $\varphi^{\succ}$ is globally conversely consistent if and only if $\succ$ is unit acyclic.

Proof.

PART I: Assume that priority structure $\succ$ has a unit cycle. We prove that $\varphi^{\succ}$ is not globally conversely consistent. Let $x, y \in X$ and (without loss of generality) $1,2,3 \in \bar{N}$ constitute a unit cycle. Let $N=\{1,2,3\}, q_{x}=q_{y}=q_{z}=1$, and $q_{x^{\prime}}=0\left(x^{\prime} \in X \backslash\{x, y, z\}\right)$. One easily verifies that $x, y$ and $1,2,3$ constitute a local cycle for $(N, q)$ (notice that the c-scarcity condition is vacuously satisfied). Hence, by Corollary $3, \varphi^{\succ}$ is not locally conversely consistent for $(N, q)$. So, $\varphi^{\succ}$ is not globally conversely consistent.

PART II: Assume $\varphi^{\succ}$ is not globally conversely consistent. Then, for some set of agents $N \subseteq \bar{N}$ and quota vector $q, \varphi^{\succ}$ is not locally conversely consistent for $(N, q)$. By Theorem 2 , the priority structure $\succ$ has a local shift for $(N, q)$. Let $x, y, z \in X\left(q_{x}, q_{y}, q_{z} \neq 0\right)$ and (without loss of generality) $1,2,3 \in N$ constitute a local shift for $(N, q)$. Consider $\tilde{q}$ with $\tilde{q}_{x}=\tilde{q}_{y}=\tilde{q}_{z}=1$ and $\tilde{q}_{x^{\prime}}=0\left(x^{\prime} \in X \backslash\{x, y, z\}\right)$. Then, $\tilde{q}$ is a unit quota vector and $x, y, z \in X$ and $1,2,3 \in N$ constitute a local shift for $(N, \tilde{q})$. By Lemma 2 (or Lemma 3 ), there is a local cycle for $(N, \tilde{q})$. Hence, the priority structure $\succ$ has a unit cycle.

Corollary 5. Let $\succ$ be a priority structure. Then, $\varphi^{\succ}$ is globally conversely consistent if and only if $\varphi^{\succ}$ is globally consistent. 


\section{References}

Balinski, M., and T. Sönmez (1999): A Tale of Two Mechanisms: Student Placement, Journal of Economic Theory 84, 73-94.

Ehlers, L. (2002): Coalitional Strategy-Proof House Allocation, Journal of Economic Theory $105,298-317$.

Ehlers, L., and B. Klaus (2003): Resource-Monotonic House Allocation, International Journal of Game Theory 32, 545-560.

Ehlers, L., and B. Klaus (2006): Efficient Priority Rules, Games and Economic Behavior $55,372-384$.

Ehlers, L., and B. Klaus (2007): Consistent House Allocation, Economic Theory 30, 561-574.

Ehlers, L., B. Klaus, and S. Pápai (2002): Strategy-Proofness and Population-Monotonicity for House Allocation Problems, Journal of Mathematical Economics 83, 329-339.

Ergin, H. İ. (2000): Consistency in House Allocation Problems, Journal of Mathematical Economics 34, 77-97.

Ergin, H. İ. (2002): Efficient Resource Allocation on the Basis of Priorities, Econometrica $70,2489-2497$.

Gale, D., and L.S. Shapley (1962): College Admissions and the Stability of Marriage, The American Mathematical Monthly 69, 9-15.

Kesten, O. (2006): On Two Competing Mechanisms for Priority-Based Allocation Problems, Journal of Economic Theory 127, 155-171.

Kojima F., and M. Utku Ünver (2011): The "Boston" School-Choice Mechanism (Version: October 8, 2011). Boston College. Mimeo.

Özkal-Sanver, İ (2013): Minimal Converse Consistent Extension of the Men-Optimal Solution, Social Choice and Welfare 40, 89-99.

Roth, A.E. (1982): The Economics of Matching: Stability and Incentives, Mathematics of Operations Research 7, 617-628.

Roth, A.E. (1984): The Evolution of the Labor Market for Medical Interns and Residents: a Case Study in Game Theory, Journal of Political Economy 92, 991-1016.

Roth, A.E. (1985): The College Admissions Problem is not Equivalent to the Marriage Problem, Journal of Economic Theory 36, 277-288.

Roth, A.E., and M.A.O. Sotomayor (1990): Two-Sided Matching: A Study in Game-Theoretic Modeling and Analysis. Econometric Society Monograph Series. New York: Cambridge University Press. 
Sasaki, H., and M. Toda (1992): Consistency and Characterization of the Core of Two-Sided Matching Problems, Journal of Economic Theory 56, 218-227.

Thomson, W. (2009): Consistent Allocation Rules (Version: June 8, 2009). University of Rochester. Mimeo.

Thomson, W. (2011): Consistency and its Converse: an Introduction. Review of Economic Design 15, 257-291.

Thomson, W. (2012): On the Computational Implications of Converse Consistency (Version: March 25, 2012). University of Rochester. Mimeo.

Thomson, W., and L. Zhou (1993): Consistent Solutions in Atomless Economies, Econometrica $61,575-587$.

Velez, R. (2012): Consistent Strategy-proof Assignment by Hierarchical Exchange (Version: December 3, 2012). Texas A\&M University. Mimeo. 